\title{
Fiabilidad y validez de la Escala del Contínuum de Salud Mental (MHC-SF) en el contexto ecuatoriano
}

\section{Reliability and validity of the Mental Health Continuum (MHC-SF) in the Ecuadorian contexts}

\author{
Eva Karina Peña Contreras ${ }^{1}$ \\ Sandra Eugenia Lima Castro ${ }^{2}$ \\ Gladys Alexandra Bueno Pacheco ${ }^{3}$ \\ Mónica Elisa Aguilar Sizer ${ }^{4}$ \\ Corey Lee M. Keyes ${ }^{5}$ \\ William Paúl Arias Medina ${ }^{6}$ \\ 1, 2, 3, 4, ${ }^{6}$ Facultad de Psicología, Universidad de Cuenca. Ecuador \\ ${ }^{5}$ Department of Sociology, Emory College. U.S.A.
}

\begin{abstract}
Resumen: El presente estudio tiene como objetivo estudiar la estructura factorial, fiabilidad y validez del Contínuum de Salud Mental (MHC-SF) de Keyes (2009) en el contexto ecuatoriano. La escala fue aplicada a dos muestras, una de 100 personas que funcionó como piloto y otra de 550 personas residentes en la ciudad de Cuenca- Ecuador, a quienes también se aplicó una adaptación del Perfil PERMA de Butler y Kern (2016) para comprobar la validez convergente y discriminante. Se observó que la aplicación en esta población presenta adecuados niveles de ajuste y alta consistencia interna $(\alpha=.91)$ y un ajuste general adecuado de la estructura de tres factores: bienestar psicológico, bienestar social, bienestar emocional propuestos por el autor, dando valores de $R M S E A=.045, C F I=$ $.996, \chi 2(52)=109.048(p=.000), G F I=.997$ y $T L I=0.993$. El modelo bifactorial presenta valores de $R M S E A=.042, C F I=.996, \chi 2(52)=103.040(p=.000), G F I=.997, T L I=0.993$. Se concluye que la versión en español de MHC-SF (Keyes, 2009) y la versión bifactorial son herramientas apropiadas para medir la salud mental en el contexto ecuatoriano.
\end{abstract}

Palabras clave: bienestar, propiedades psicométricas, salud mental, evaluación psicológica, desarrollo instrumental, MHC-SF

\begin{abstract}
The present study aims to study the factor structure, reliability and validity of the Mental Health Continuum (MHC-SF) of Keyes (2009) in the Ecuadorian context. The scale was applied to two samples, one of 100 people that worked as a pilot and another of 550 people resident in the city of Cuenca-Ecuador, who also were applied an adaptation of the PERMA Profile of Butler and Kern (2016) to verify the convergent and discriminant validity. It was observed that the application in this population presents adequate levels of adjustment and high internal consistency $(\alpha=.91)$ and an adequate general adjustment of the structure of three factors: psychological well-being, social welfare and emotional well-being proposed by the author whose main fit values are $R M S E A=.045$, $C F I=.996, \chi 2(52)=109.048(p=.000), G F I=.997$ y $T L I=0.993$. The bifactor model presents good values as well: $R M S E A=.042, C F I=.996, \chi 2(52)=103.040(p=.000), G F I=.997, T L I=0.993$. We conclude that the Spanish version of MHC-SF (Keyes, 2009) and the bifactor model are appropriate tools to measure mental health in the Ecuadorian context.
\end{abstract}

Key Words: wellbeing, psychometric properties, mental health, psychological assessment, instrumental development, MHC-SF

Cómo citar este artículo:

Peña Contreras, E. K., Lima Castro, S. E., Bueno Pacheco, G. A., Aguilar Sizer, M. E., Keyes, C. L. M., \& Arias Medina, W. P. (2017). Fiabilidad y validez de la Escala del Contínuum de Salud Mental (MHC-SF) en el contexto ecuatoriano. Ciencias Psicológicas, 11(2), 223-232. doi: https://doi.org/10.22235/cp.v11i2.1499

La Escala del Contínuum de Salud Mental (MHC-SF) forma parte de un proyecto más amplio: "Variables asociadas al Bienestar de Personas con y sin Discapacidad", con apoyo y financiamiento de la Dirección de Investigación de la Universidad de Cuenca, Ecuador (Mauricio Espinoza, Ph.D., Director), con asesoría de José Luis Vilches , Ph.D.

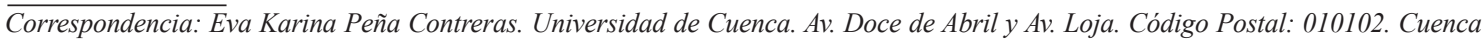
- Ecuador, e-mail: eva.pena@ucuenca.edu.ec; Sandra Eugenia Lima Castro, e-mail: sandra.lima@ucuenca.edu.ec; Gladys Alexandra Bueno Pacheco, e-mail: alexandra.bueno@ucuenca.edu.ec; Mónica Elisa Aguilar Sizer, e-mail: monica.aguilars@ucuenca.edu.ec; Universidad de Cuenca. Corey Lee M. Keyes, e-mail: ckeyes@emory.edu, Emory College. William Paúl Arias Medina, e-mail: paul. arias@ucuenca.edu.ec, Universidad de Cuenca. 


\section{Introducción}

La dimensión positiva de la salud ha sido destacada por la OMS (2016) como "un estado de completo bienestar fisico, mental y social, y no solamente la ausencia de afecciones o enfermedades" concepto que a lo largo de los años ha incorporado constructos que van desde el modelo médico de presencia o ausencia de enfermedad, hasta el resaltar las capacidades y potencialidades del ser humano como herramientas de crecimiento personal, lo que según Barrera y Flores (2015) ha dado como resultado una ampliación de la visión hacia una mayor complejidad en torno a la conceptualización y al acercamiento teórico.

Tradicionalmente las investigaciones en el área de la psicología han estado enfocadas a los problemas de la salud, pero en la actualidad sabemos que esta orientación no proporciona una evaluación completa de la salud mental de un individuo. Por ello en los últimos años ha existido un crecimiento en el interés por el funcionamiento general del ser humano y de la salud mental en particular (Kokko, Korkalainen, \& Lyyra, 2013). Además, los estudios han demostrado que el funcionamiento positivo en la salud mental esta asociado al bienestar psicológico y al bienestar social, dejando ver las ventajas de su estudio a lo largo del ciclo vital y con ello las investigaciones de la psicología positiva y el bienestar se han visto fortalecidas (Keyes, 2005).

El estudio del bienestar en el área de la psicología se inicia en dos grandes líneas, la hedónica y eudaimónica (Keyes, Corey, Shmotkin, \& Ryff, 2002). La primera representada por la investigación enfocada al estudio del bienestar subjetivo, indicador de la valoración que las personas tienen respecto a la satisfacción con la vida, la felicidad y las capacidades afectivas positivas, y la segunda orientada al bienestar psicológico, concepto que incluye las dimensiones sociales, subjetivas y psicológicas, además de comportamientos relacionados con la salud en general que responden al funcionamiento positivo del ser humano. Estos autores indican que el bienestar subjetivo y el psicológico están relacionados, y aunque sus dimensiones difieren en ciertos aspectos, existe aparentemente un cierto solapamiento entre algunos constructos como, por ejemplo: autoaceptación que corresponde al modelo del bienestar subjetivo y dominio del entorno que pertenecería al modelo del bienestar psicológico y que responde a la habi- lidad de las personas para crear o elegir ambientes que favorecen la satisfacción de sus necesidades y deseos (Blanco \& Diaz, 2005) pueden sobreponerse u ocultarse la una en la otra cuanto se trata de observarlas.

Podemos observar que las teorías se complementan, y aunque cada teoría fundamenta el hecho de que las dimensiones son exclusivas, consideramos importante que además de las dos líneas tradicionales de estudio del bienestar resulta necesario inscribir al ser humano en un marco social (Blanco \& Diaz, 2005). Por lo tanto se demuestra la utilidad de los modelos multidimensionales del bienestar que integran teorías y componentes tanto de la perspectiva hedónica como la eudaimónica (Huppert \& So, 2013; Keyes, 2007).Es así que la propuesta del modelo multidimensional de Keyes (2007) de salud mental positiva, destaca al integrar, el bienestar emocional: que se constituye de los afectos, positivo alto y negativo bajo, el bienestar psicológico, compuesto por auto-aceptación, crecimiento personal, propósito en la vida, dominio del entorno, autonomía personal y relaciones positivas con los demás y el bienestar social constituido por la aceptación social percibida por el sujeto, actualización social (en donde el sujeto sano confía en el futuro de la sociedad, en su potencial de crecimiento de desarrollo y su capacidad para producir bienestar), la contribución social, cohesión social e integración social.

Este modelo teórico fue la base para desarrollar la escala Mental Health Continuum Long Form, MHC-LF., que en primera instancia estuvo conformada por 40 ítems y era un cuestionario de autoregistro. (Keyes, 2002; 2005; 2007). Posteriormentese desarrolló una versión más corta, el Mental Health Continuum Short Form [contínuum de salud mental] (MHC-SF) (Keyes, 2009) con 14 ítems esta versión se popularizó rápidamente por su brevedad en la aplicación conservando su carácter de autoregistro.

El MHC-SF ha sido traducido a diferentes idiomas y validado a través de diversos contextos culturales (Carvalho, Salgado, Marquéz, \& Marocó, 2016; Joshanloo, Wissing, Khumalo, \& Lamers, 2013; Karaś, Cieciuch, \& Keyes, 2014; Petrillo, Capone, Caso, \& Keyes, 2015), confirmándose la estructura tri factorial en la mayoría de estudios, aunque en las adaptaciones serbia y sudafricana el modelo no se replica y aparece como modelo bifactorial (du Plessis \& de Bruin, 2015; Jovanović, 2015) el cuál es explicado como una so- 
lución razonable para escalas multidimensionales como el MCH-SF (Chen, West, \& Sousa, 2006).

En cuanto a la fiabilidad de la versión original del MHC-SF, la escala ha demostrado una consistencia interna medida por el alfa de Cronbach siempre superior a.80 en adolescentes (edades 1218) y adultos en los Estados Unidos, en los Países Bajos y en Sudáfrica (Keyes, 2005; 2009; Lamers, Glas, Westerhof, \& Bolemeijer, 2011). La fiabilidad test-retest del MHC-SF durante tres períodos sucesivos de 3 meses promedió .68 y el test-re test de 9 meses fue de .65 (Lamers et al., 2011). La estructura factorial se conservó en las dos formas de la escala (larga y corta) con su modelo de tres factores: bienestar emocional, psicológico y social.

Para examinar la validez divergente del test se ha correlacionado el resultado del cuestionario Brief Symptom Inventory [Inventario corto de síntomas de enfermedad mental] con el MHC-SF dando como resultado correlaciones de - .34 (Lamers et al., 2011). Adicionalmente, se ha analizado el MHC-SF conjuntamente con los cuestionarios Positive and Negative Affect Schedule [Lista de afectos positivos o negativos], (PANAS PN), General Health Questionnaire [Cuestionario de salud metal general] (GHQ) observándose una correlación negativa en las sub escalas de afecto negativo en un rango moderado entre $-.33 \mathrm{y}$ -.45 (Petrillo, Capone, Caso, \& Keyes, 2015).

Varios estudios de adaptación y validación de la escala MHC-SF han sido realizados demostrando en general buenos niveles de ajuste. En China se realizó un estudio que buscaba verificar la fiabilidad interna, la estructura dimensional e invariancia entre grupos y la presencia de efectos techo y suelo de la misma que fue aplicada a una muestra de 5.399 personas, este estudio implicó la traducción del cuestionario mediante un equipo de investigación de China, y utilizaron el método de retro traducción aplicada en un estudio piloto con una muestra de 285 estudiantes, mediante análisis factorial confirmatorio se ratificaron los tres factores sugeridos en la escala original y la fiabilidad indicó un alfa de Cronbach de .80 indicando buena consistencia, y que los efectos techo y suelo eran insignificantes (Guo et al., 2015).

La escala también ha sido adaptada en varios contextos culturales como Italia (Petrillo, Capone, Caso, \& Keyes, 2015), Polonia (Karaś, Cieciuch, \& Keyes,2014), Portugal (Carvalho, Salgado, Marquéz, \& Maroco, 2016), Holanda, en Irán y
Sudáfrica en donde los valores de los alfas de Cronbach han oscilado entre .86 y.95 reiterando en la mayoria de ellos la tridimensionalidad del modelo y ajustes adecuados en el modelo factorial (Joshanloo, Wissing, Khumalo, \& Lamers, 2013). En cuanto a países latinoamericanos el MHC-SF se tradujo al español y se aplicó a una muestra de 3.335 adultos en Chile y mediante análisis factorial probaron los modelos de tres y dos factores. Aunque obtuvieron un ajuste apropiado en el primero, en el segundo modelo mostró un ajuste superior. El alpha de Cronbach obtenido fue de .94 mostrando alta confiabilidad (Echeverría, Torres, Pedrals, Padilla, Rigotti, \& Bitran, 2017). En Argentina se estudiaron las propiedades psicométricas de la escala en una muestra de 1.300 adultos mediante análisis factorial confirmatorio, validación cruzada, invariancia factorial y correlaciones con criterios externos, en donde los resultados indicaron un ajuste adecuado al modelo de tres factores propuesto por Keyes (Keyes, 2005; 2007; 2009) e invariable a través del sexo y la edad, la consistencia interna obtenida fue de .89 con el alpha de Cronbach, resultados que apoyan al modelo del continuum de salud mental (Lupano Perugini, de la Iglesia, Castro Solano, \& Keyes, 2017).

En cuanto a la validez de constructo se han utilizado diversas escalas: calidad de vida (MMQL) y depresión (HADS), (Guo et al., 2015), Cronograma de afecto positivo PANAS (Petrillo, Capone, Caso, \& Keyes, 2015), y Escala de Satisfacción con la vida (SWLS) (Joshanloo, Wissing, Khumalo, \& Lamers, 2013).

Considerando que en Ecuador no se cuenta con instrumentos que evalúen la salud mental positiva, si bien actualmente existen versiones en español de la escala MHC-SF, es necesario adaptar el instrumento a nuestro medio local debido a que el lenguaje español posee particularidades idiomáticas asociadas a los distintos países y regiones en los que se habla. El objetivo del presente estudio fue realizar la adaptación psicométrica y aportar datos de validez convergente y discriminante, $y$ fiabilidad de la versión en español para el contexto ecuatoriano, de escala del contínuum de salud mental (MHCSF).El principal aporte de este estudio es contar con herramientas necesarias adaptadas al contexto para obtener datos confiables en la evaluación de la salud mental positiva facilitando la acumulación de conocimiento válido en un campo crecientemente importante de la psicología. 


\section{Método}

\section{Instrumentos}

Encuesta sobre datos sociodemográficos: fue elaborada por el equipo de investigación y recoge datos de edad, sexo, nivel educativo, ocupación actual, ingresos económicos. La misma fue aplicada en los dos estudios.

- Escala Mental Health Contínuum Short Form: Se elaboró una versión en español de la escala. Se siguieron las normas generales de traducción y adaptación de test (Guillemin, Bombardier, \& Beaton, 1993), incluyendo la retro-traducción (Brislin, Lonner, \& Thorndike, 1973), considerando que para la fecha de elaboración de esta investigación aún no se publicaban estudios de adaptación o validación de la escala al español.

Se efectuó un estudio piloto y posteriormente se aplicó el instrumento a una segunda muestra para el análisisde sus propiedades psicométricas. La forma corta consiste en 14 ítems que responden a una escala Likert de uno a seis puntos, siendo 1 nunca y 6 todos los días, teóricamente 3 ítems pertenecen a la dimensión bienestar emocional, 5 bienestar social, y 6 dimensión de bienestar psicológico (Keyes, 2009).

- Perma Profiler de Buttler y Kern (2016): versión adaptada al español (Lima-Castro et al., 2017). Esta escala está compuesta por 23 ítems, 15 de ellos evalúan el bienestar, y tres factores independientes que son salud, emociones negativas y soledad. Esta escala tipo Likert de 11 puntos posee valores que van desde 0 (nunca) a 10 (siempre) o 0 (nada) a 10 (completamente). Esta escala reporta propiedades psicométricas adecuadas y fue utilizada únicamente en el segundo estudio.

\section{Estudio 1}

\section{Muestra 1}

En esta fase de estudio piloto la muestra estuvo constituida por 100 personas, $49 \%$ de población masculina $(n=49)$ y $51 \%$ de población femenina $(n=51)$ con edades comprendidas entre los 20 y 86 años, la edad media de los sujetos fue de 43.88 años y desviación estándar de 16.52 años). La muestra pertenece a la zona urbana de la ciudad de Cuenca.

\section{Procedimiento}

La muestra de esta primera parte del estudio fue recogida por visita domiciliaria, mediante el método a conveniencia durante los meses de mayo y junio del año 2016. Se solicitó un consentimiento informado y se aplicó además del cuestionario una encuesta de factores sociodemográficos. Se solicitó a cada participante información acerca de las dificultades que encontraban en responder a cada una de las preguntas.

\section{Resultados}

En el estudio piloto se pudo observar que el $38 \%$ de la población $(n=46)$ tuvo dificultades en el ítem número 7 ("Que las personas son básicamente buenas"), el mismo no era comprendido, por lo que se recurre a la versión en inglés para una retro-traducción y se decide re-estructurarlo a "Que las personas son buenas". Cabe recalcar que la modificación fue justificada por los participantes y las características culturales de la población. Se modificó también la disposición en el diseño de la prueba, con el fin de evitar confusiones. Por tratarse de una escala tipo Likert, los números se colocaron debajo de cada pregunta en lugar del costado derecho.

\section{Estudio 2 \\ Muestra 2}

La muestra fue tomada por conveniencia considerando un universo de 315839 personas con edades entre 19 y 89 años pertenecientes a la ciudad de Cuenca, considerando las diferentes características sociodemográficas de la población, e involucró la participación de 562 personas $\left(M_{\mathrm{Edad}}=42.31\right.$ años, $\left.D E_{\text {Edad }}=4.26\right)$, de los cuales el $27.4 \%$ eran hombres $(n=154)$ y $72.6 .2 \%$ mujeres $(n=408)$, todos ecuatorianos, residentes de la ciudad de Cuenca. Respecto al nivel educativo de los participantes, indicó sin instrucción $1.4 \%(n=8)$, sin bachillerato $8.4 \%(n=47)$, con bachillerato $22.1 \%(n=124)$, con estudios universitarios $67.6 \%(n=380)$, no contesta el 3\% $(n=3)$. En cuanto al estado civil se registró soltero $50.2 \%(n=282)$, casado $32.4 \%(n=182)$, unión libre $1.8 \%(n=10)$, separado $0.9 \%(n=5)$, divorciado $6.8 \%(n=38)$ y viudo $7 \%(n=41)$, no contesta. $7 \%(n=4)$.

Las aplicaciones de los reactivos se realizaron desde los meses de agosto y septiembre del año 2016, y se contó con participación voluntaria de personas pertenecientes a la Universidad del Azuay, Cuenca, rehabilitación física del centro de atención a jubilados y familiares de pacientes que se encon- 
traban en centros de atención de salud, y centro de atención al adulto mayor del Instituto de Seguridad Social (IESS), a quienes se les aplicó a la vez el Perfil Perma y una encuesta sociodemográfica, bajo la firma de un consentimiento informado.

\section{Procedimiento}

La escala y la encuesta fueron administradas por los autores de este trabajo. Se obtuvieron los permisos respectivos de las instituciones que participaron en la investigación. Como parte del proceso de preparación a los participantes, se informó acerca de los objetivos del estudio y se instruyó a los adultos acerca del cuestionario a realizar, se aplicó a la vez una encuesta para recolectar datos sociodemográficos. La participación fue totalmente voluntaria, se mantuvo el carácter de la escala de autorregistro pero con la debida guía y apoyo a los participantes de manera que todas las preguntas sean contestadas de ser posible. Cada persona se demoró alrededor de 20 minutos en responder el cuestionario.

\section{Análisis de datos}

En primera instancia, se evalúan los estadisticos descriptivos de las variables de la escala, principalmente lo que concierne a asimetría, curtosis, correlaciones ítem-test y la distribución de las variables. Se eliminan 12 observaciones ya que a pesar de la orientación de los encuestadores, los formularios no habían sido completados en su totalidad.

Se realiza un análisis factorial exploratorio (AFE) para determinar el número de dimensiones a considerarse para, a continuación proseguir con un análisis factorial confirmatorio (AFC).

Para evaluar la estructura factorial de la escala se parte de un modelo de ecuaciones estructurales con matrices policóricas con estimador DWLS (Diagonally Weighted Least Squares) robusto que es el método recomendado para muestras pequeñas o medianas (Rhemtulla, Brosseau-Liard, \& Savalei, 2012).Como se realiza en otros estudios previos, esta investigación se plantea la hipótesis de que la escala posee tres factores latentes correlacionados entre sí y posteriormente comprueba un modelo bifactorial con tres dimensiones específicas y una general.

Para evaluar los resultados se utilizaron diversos indices de ajuste, como chi cuadrado; $C F I$ [índice de ajuste comparativo] y $T L I$ [índice de Tucker \& Lewis] cuyos valores entre .90 a .95 indican ajuste aceptable del modelo mientras que valores mayores a .96 indican buen ajuste $(\mathrm{Hu} \&$ Bentler, 1999). Además, se utiliza la RMSEA, cuyo valor es considerado aceptable cuando es inferior a .08 y bueno cuando es menor a .05 (Steiger \& Lind, 1980). Los modelos antes descritos se evaluan utilziando el software FACTOR.

Además de estudiar la validez factorial se evaluó la fiabilidad de la escala mediante el alpha de Cronbach y el omega de McDonald, teniendo en cuenta que en el caso del primero los valores de .70 a .80 son considerados como aceptables, y sobre los .80 se considera de alta fiabilidad (Cicchetti, 1994). Por otro lado, es importante señalar que el omega de McDonald es útil cuando las muestras son grandes, preferiblemente mayores a 1000 observaciones (Ten Berge \& Socan, 2004).

Una vez confirmada la estructura factorial de la escala original,con modificaciones cualitativas de la fase de estudio piloto, se compararon las variables sociodemográficas para evaluar los resultados y diferencias entre las categorías de las variables. Se realizaron pruebas de correlación de Spearman, pruebasde normalidad de Kolmogorov-Smirnov, prueba de suma de rangos de Wilcoxon, prueba de Kruskal-Wallis y el correspondiente test post-hoc de Dunn.

\section{Resultados}

\section{Validez factorial}

Los estadísticos descriptivos de las variables de la escala muestran que los datos no siguen una distribución normal, lo cual se observa inicialmente por la asimetría y curtosis y es posteriormente corroborado con la prueba de Kolmogorov-Smirnov. Las correlaciones ítem-test son positivas y con magnitud moderada o fuerte. Los datos se muestran en las tabla 1.

Respecto a la adecuación de la matriz de correlación se obtiene un estadístico de Barlett de $3747.4(d f=91 ; p=.000)$ mientras que el test KaiserMeyer-Olkin muestra un valor de .9395 considerado como muy bueno. Estos dos valores indican que la matriz es factorizable.

El análisis factorial exploratorio sugiere la presencia de un factor por lo que se considera evaluar un modelo bifactorial con tres dimensiones específicas y una general además de la estructura original de tres factores, tal como se presenta en la figura 1 . 
Tabla 1.

Estadísticos descriptivos de los items del Contínuum de Salud Mental (MHC-SF)

\begin{tabular}{ccccccccc}
\hline Variable & Media & $\begin{array}{c}\text { Intervalo de } \\
\text { Confianza (95\%) }\end{array}$ & Varianza & Asimetría & $\begin{array}{c}\text { Curtosis } \\
\text { (Centrada) }\end{array}$ & $\begin{array}{c}\text { Correlación } \\
\text { item-test }\end{array}$ & \multicolumn{2}{c}{ Kolmogorov-Smirnov } \\
\cline { 6 - 9 } Estadístico & Sig. \\
\hline be1 & 3.672 & $(3.58-3.76)$ & .711 & -.612 & .32 & .649 & .311 & .000 \\
be2 & 4.091 & $(3.99-4.19)$ & .772 & -.885 & .735 & .722 & .240 & .000 \\
be3 & 4.033 & $(3.93-4.13)$ & .823 & -.868 & .56 & .772 & .257 & .000 \\
bs1 & 3.813 & $(3.71-3.92)$ & .925 & -.673 & -.028 & .676 & .279 & .000 \\
bs2 & 3.755 & $(3.64-3.87)$ & 1.111 & -.617 & -.368 & .664 & .256 & .000 \\
bs3 & 3.579 & $(3.47-3.69)$ & .995 & -.356 & -.477 & .625 & .235 & .000 \\
bs4 & 3.392 & $(3.29-3.50)$ & .906 & -.132 & -.599 & .609 & .222 & .000 \\
bs5 & 3.216 & $(3.11-3.32)$ & .993 & -.112 & -.627 & .620 & .208 & .000 \\
bp1 & 3.936 & $(3.84-4.03)$ & .796 & -.796 & .622 & .759 & .277 & .000 \\
bp2 & 4.067 & $(3.97-4.17)$ & .81 & -.746 & .193 & .694 & .229 & .000 \\
bp3 & 3.806 & $(3.71-3.90)$ & .792 & -.678 & .404 & .715 & .288 & .000 \\
bp4 & 4.06 & $(3.97-4.15)$ & .706 & -.959 & 1.383 & .580 & .275 & .000 \\
bp5 & 3.889 & $(3.79-3.99)$ & .861 & -.803 & .45 & .693 & .285 & .000 \\
bp6 & 4.16 & $(4.06-4.26)$ & .813 & -1.049 & .904 & .768 & .248 & .000 \\
\hline
\end{tabular}

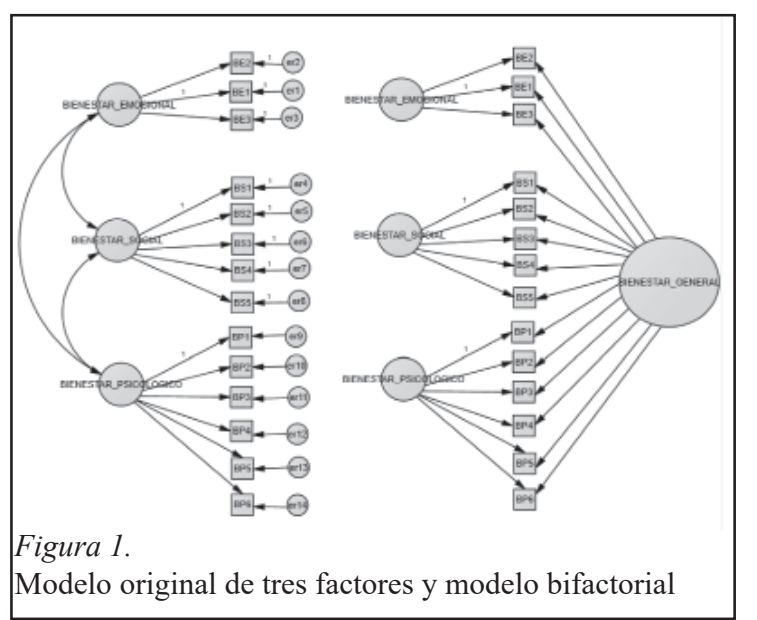

El análisis factorial confirmatorio (AFC) para la escala con los tres factores propuestos por el autor (Keyes, 2002) muestra un ajuste general adecuado dando usando el método de DWLS y utilizando matrices de correlación policóricas se obtenien los siguientes valores: $R M S E A=.045, C F I=.996, \chi^{2}(52)$ robusto con media y varianza ajustadas $=109.048$ $(p=.000), G F I=.997, T L I=0.993$ Las cargas factoriales se observan en la tabla 2.

Adicionalmente, un análisis factorial confirmatorio (AFC) del modelo bifactorial reportado en otro estudio (Echeverría et al, 2007) muestra valores de $R M S E A=.042, C F I=.996, \chi^{2}$ (52) robusto con media y varianza ajustadas $=103.040$ ( $p=.000), G F I=.997, T L I=0.993$.

La matriz de cargas factoriales se presenta en la tabla 3.

Se calcula el coeficiente de correlación de Spearman entre las 3 dimensiones de bienestar cuyos resultados se presentan en la tabla 4.
Tabla 2.

Cargas factoriales del modelo original de tres factores

\begin{tabular}{cccc}
\hline Variable & $\begin{array}{c}\text { Bienestar } \\
\text { emocional }\end{array}$ & $\begin{array}{c}\text { Bienestar } \\
\text { social }\end{array}$ & $\begin{array}{c}\text { Bienestar } \\
\text { psicológico }\end{array}$ \\
\hline be1 & .149 & & \\
be2 & .192 & & \\
be3 & .05 & & \\
bs1 & & .06 & \\
bs2 & & .14 & \\
bs3 & & .597 & \\
bs4 & & .634 & \\
bs5 & & .9 & \\
bp1 & & & .884 \\
bp2 & & & .851 \\
bp3 & & & .674 \\
bp4 & & & .224 \\
bp5 & & & .658 \\
bp6 & & & .828 \\
\hline
\end{tabular}

Tabla 3.

Cargas factoriales para el modelo bifactorial

\begin{tabular}{ccccc}
\hline Variable & $\begin{array}{c}\text { Bienestar } \\
\text { emocional }\end{array}$ & $\begin{array}{c}\text { Bienestar } \\
\text { social }\end{array}$ & $\begin{array}{c}\text { Bienestar } \\
\text { psicológico }\end{array}$ & $\begin{array}{c}\text { Bienestar } \\
\text { general }\end{array}$ \\
\hline be1 & .085 & & & .629 \\
be2 & .103 & & & .731 \\
be3 & .017 & & & .764 \\
bs1 & & .029 & & .669 \\
bs2 & & .095 & & .67 \\
bs3 & & .414 & & .578 \\
bs4 & & .452 & & .549 \\
bs5 & & .629 & & .569 \\
bp1 & & & .373 & .756 \\
bp2 & & & .354 & .692 \\
bp3 & & & .28 & .683 \\
bp4 & & & .095 & .585 \\
bp5 & & & .267 & .679 \\
bp6 & & & .35 & .795 \\
\hline
\end{tabular}


Tabla 4.

Coeficientes de correlación de Spearman entre las tres dimensiones de bienestar

\begin{tabular}{cccc}
\hline & $\begin{array}{c}\text { Bienestar } \\
\text { social }\end{array}$ & $\begin{array}{c}\text { Bienestar } \\
\text { psicológico emocional }\end{array}$ \\
\hline Bienestar social & 1 & & \\
Bienestar psicológico & $.627 *$ & 1 & \\
Bienestar emocional & $.593^{*}$ & $.668^{*}$ & 1 \\
\hline *significativo a .01 & & &
\end{tabular}

\section{Fiabilidad}

En cuanto a la fiabilidad del modelo como lo propone Keyes (2009), el alfa de Cronbach presenta un valor global que indica alta consistencia interna para el total de la escala MHC-SF $(\alpha=.91)$. El coeficiente de consistencia interna para la sub-escala Bienestar Emocional $(\alpha=.80)$ se valora como alto, siendo el ítem más representativo "me he sentido satisfecho con la vida"; la sub-escala Bienestar Social muestra un valor moderado $(\alpha=.72)$, su ítem más representativo es "me he sentido que pertenezco a una comunidad, grupo, escuela o vecindario"; finalmente, la sub-escala Bienestar psicológico presenta un alfa considerado alto $(\alpha=.85)$, cuyo ítem más representativo es "me he sentido seguro de expresar mis propias ideas y opiniones".

Las correlaciones inter ítem promedio se presentan en la tabla 5. El omega de McDonald alcanza un valor de .933 aunque se recomienda su uso en muestras de al menos 1000 observariones (Ten Berge \& Socan, 2004).

Adicionalmente, se calcula el coeficiente de correlación de Pearson con la sub-escala Emociones Negativas de la escala Perma Profiler de Buttler y
Kern (2016), versión adaptada al español (LimaCastro et al., 2017), la que reporta una correlación moderada ( $r=.30 ; p=.01)$ sugiriendo que a mayor salud mental positiva, menor grado de emociones negativas.

Se realiza la prueba de suma de rangos Wilcoxon ( $U$ de Mann-Whitney) para dos muestras comparando el bienestar emocional, social y psicológico entre hombres y mujeres. Además, se realiza el test de Kruskal Wallis para más de dos grupos y el posterior test post-hoc de Dunn. Estadísticos descriptivos y resultados de las pruebas de hipótesis se presentan en las tablas 6 y 7 .

\section{Discusión}

El presente estudio evaluó las características psicométricas de la prueba Mental Health Continuum Short Form (MHC-SF) de Keyes (2009) que es un cuestionario de auto reporte elaborado para medir la salud mental positiva, con tres sub-escalas de bienestar: emocional, psicológica y social. Está compuesta por 14 ítems y fue aplicado en primera instancia a una población por conveniencia de 100 personas para un estudio piloto en donde se revisó la validez de contenido en una aplicación grupal de la escala, lo cual dio como resultante modificaciones de tipo semántico y a nivel de consigna de la escala. En un segundo estudio se aplica a una población de 550 participantes en una muestra por conveniencia. Las dos muestras se tomaron en la ciudad de Cuenca, Ecuador. La validez de la estructura fue probada empleando el análisis factorial exploratorio (AFE) que sugiere la presencia de un factor, por lo que se

Tabla 5.

Evaluación de la confiabilidad del Contínuum de Salud Mental (MHC-SF)

Correlación interítem promedio: .424

\begin{tabular}{|c|c|c|c|c|c|c|c|c|c|c|c|c|c|c|}
\hline & be1 & be2 & be3 & bs1 & bs2 & bs3 & bs4 & bs5 & bp1 & bp2 & bp3 & bp4 & bp5 & bp6 \\
\hline be1 & 1 & & & & & & & & & & & & & \\
\hline be 2 & .523 & 1 & & & & & & & & & & & & \\
\hline be 3 & .551 & .650 & 1 & & & & & & & & & & & \\
\hline bs 1 & .363 & .508 & .456 & 1 & & & & & & & & & & \\
\hline bs 2 & .380 & .408 & .427 & .528 & 1 & & & & & & & & & \\
\hline bs 3 & .350 & .362 & .405 & .342 & .395 & 1 & & & & & & & & \\
\hline bs 4 & .339 & .362 & .432 & .320 & .321 & .440 & 1 & & & & & & & \\
\hline bs 5 & .354 & .286 & .378 & .341 & .394 & .529 & .515 & 1 & & & & & & \\
\hline bp1 & .458 & .544 & .582 & .473 & .393 & .389 & .379 & .384 & 1 & & & & & \\
\hline bp2 & .348 & .472 & .525 & .406 & .311 & .367 & .338 & .374 & .596 & 1 & & & & \\
\hline bp3 & .438 & .451 & .524 & .406 & .385 & .365 & .437 & .418 & .569 & .516 & 1 & & & \\
\hline bp4 & .338 & .385 & .363 & .407 & .432 & .247 & .224 & .232 & .378 & .358 & .318 & 1 & & \\
\hline bp5 & .398 & .417 & .492 & .390 & .411 & .307 & .331 & .334 & .562 & .505 & .476 & .449 & 1 & \\
\hline bp6 & .430 & .591 & .632 & .486 & .467 & .384 & .328 & .288 & .598 & .556 & .564 & .479 & .584 & 1 \\
\hline
\end{tabular}


Tabla 6

Resultados de las dimensiones de bienestar según género y etapas de la vida adulta

\begin{tabular}{|c|c|c|c|c|c|c|c|c|}
\hline & \multirow{2}{*}{ Variable } & \multicolumn{3}{|c|}{ Bienestar Emocional } & \multicolumn{2}{|c|}{ Bienestar Social } & \multicolumn{2}{|c|}{ Bienestar Psicológico } \\
\hline & & $\mathrm{n}$ & Media & Mediana & Media & Mediana & Media & Mediana \\
\hline \multirow{3}{*}{ Sexo } & Hombre & 153 & 11.222 & 12 & 16.967 & 17 & 22.784 & 24 \\
\hline & Mujer & 397 & 12.045 & 12 & 18.103 & 19 & 24.415 & 25 \\
\hline & & & \multicolumn{2}{|c|}{$z=-3.930 ; p=.000$} & \multicolumn{2}{|c|}{$z=-2.912 ; p=.003$} & \multicolumn{2}{|c|}{$z=-4.344 ; p=.000$} \\
\hline \multirow{4}{*}{ Adultez } & Adultez temprana & 305 & 11.409 & 12 & 17.055 & 17 & 22.698 & 23 \\
\hline & Adultez intermedia & 82 & 12.036 & 12 & 18.426 & 19 & 22.698 & 25 \\
\hline & Adultez tardía & 162 & 12.450 & 13 & 18.796 & 19 & 25.839 & 27 \\
\hline & & & \multicolumn{2}{|c|}{$\chi^{2}(2)=27.874 ; p=.000$} & $\chi^{2}(2)=2$ & $76 ; p=.000$ & \multicolumn{2}{|c|}{$\chi^{2}(2)=82.077 ; p=.000$} \\
\hline
\end{tabular}

Tabla 7.

Test de Dunn para etapas de la adultez

\begin{tabular}{cllll}
\hline & & Adultez temprana & Adultez intermedia & Adultez tardía \\
\hline \multirow{2}{*}{ Bienestar Emocional } & Adultez temprana & & & \\
& Adultez intermedia & $-2.575, p=.005$ & & \\
& Adultez tardía & $-5.217, p=.000$ & $-1.378 p=.084$ & \\
& & Adultez temprana & Adultez intermedia & Adultez tardía \\
Bienestar Social & Adultez temprana & & & \\
& Adultez intermedia & $-3.378 p=.000$ & & \\
& Adultez tardía & $-5.001 p=.0000$ & $-.486 p=.313$ & \\
& & Adultez temprana & Adultez intermedia & Adultez tardía \\
& Adultez temprana & & & \\
Bienestar Psicológico & Adultez intermedia & $-4.771 p=.000$ & & \\
& Adultez tardía & $-8.754 p=.0000$ & $-1.900 p=.028$ & \\
\hline
\end{tabular}

considera evaluar el modelo bifactorial con tres dimensiones específicas y una general. Posteriormente mediante análisis factorial confirmatorio (AFC) usando el método de DWLS y utilizando matrices de correlación policóricas, se observa la estructura factorial multidimensional propuesta por el autor (Keyes, 2009) de tres factores y la validez estructural del modelo al igual que los estudios realizados en contextos polaco (Karaś et al., 2014), chino (Guo et al., 2015), italiano (Petrillo et al., 2015) etc., adicionalmente este estudio realiza un análisis factorial confirmatorio (AFC) que muestra la estructura con dos factores como los presentados en el estudio de Echeverría et al. (2017), observándose que el modelo bifactorial presenta buenos indicadores de ajuste.

En cuanto a lo que podemos concluir de la correlación entre las 3 dimensiones (Bienestar social, emocional y psicológico y social) las correlaciones son significativas, demostrando de manera empírica la propuesta teórica de Keyes (2007) en su visión multidimensional de la salud mental positiva o como se ha llamado en el estudio de Echeverria et al. (2017) Bienestar General, integrando dentro del concepto de salud mental positiva o bienestar general, en el bienestar emocional: que se constituye de los afectos, positivo alto y negativo bajo, el bienestar psicológico, compuesto por auto- aceptación, crecimiento personal, propósito en la vida, dominio del entorno, autonomía personal y relaciones positivas con los demás y el bienestar social constituido por la aceptación social percibida por el sujeto, actualización social (en donde el sujeto sano confía en el futuro de la sociedad, en su potencial de crecimiento, de desarrollo y su capacidad para producir bienestar), la contribución social, cohesión social e integración social.

En esta investigación los modelos de fiabilidad referentes al alpha de Cronbach y al omega de McDonald alcanzan valores de estimación alta de consistencia interna, lo que coincide al menos en el primer valor en la mayoría de investigaciones con los otros trabajos de adaptación y validación realizados en diferentes países en donde el modelo se ajusta de manera adecuada (Guo et al., 2015). Estos resultados garantizan el uso de esta herramienta como parte de la evaluación diagnostica e investigación en el contexto ecuatoriano.

A mayor salud mental, mayor bienestar y a mayor salud mental menores emociones negativas usando la sub-escala de Emociones Negativas de la escala Perma Profiler de Buttler y Kern (2016), lo cual coincide con resultados encontrados en otras investigaciones en donde se contraponen enfermedad mental y salud mental positiva (Petrillo et al., 2015). 
Los tres factores no siguen una distribución normal de acuerdo a las pruebas realizadas sino una distribución simétrica negativa. Las correlaciones entre los factores bienestar social, emocional y psicológico son significativas para todas las combinaciones entre los tres factores, tal y como lo señala Keyes en su modelo multidimensional del bienestar (Keyes, 2002).

En la comparación de las personas referente al sexo con relación a los tres factores, se encontró que las diferencias entre hombres y mujeres son significativas, puntuando el sexo femenino con valores más altos para las tres dimensiones, pero investigaciones referentes al bienestar en mujeres tambien han reportado que las mujeres obtienen mayores puntajes en potenciar sus propias capacidades y en desarrollo personal (Yañez \& Cárdenas, 2010).

Cuando se analizan los resultados de los individuos clasificados por edad (adultez temprana, adultez media y adultez tardía) para cada dimensión de bienestar se observa que la población en adultez tardía registra valores promedio más alto. La prueba de Kruskal-Wallis muestra que en las tres dimensiones de bienestar existe una diferencia estadísticamente significativa entre al menos un par de categorías. Mediante la prueba de Dunn se evidencia que en cuanto al bienestar emocional, la diferencia entre el valor mediano entre el grupo de adultez tardía y adultez temprana; y adultez tardía y adultez media es estadísticamente significativa, no así la diferencia existente entre adultez temprana y adultez media. Los mismos resultados se observan en la sección de bienestar social, mientras que en el bienestar psicológico se observa que todas las diferencias entre todas las categorías son estadísticamente significativas.

Las personas de la tercera edad reportan puntajes medios y medianos más altos en todas las dimensiones de bienestar estudiadas. Por un lado, se ha evidenciado que los adultos mayores acumulan sabiduría y se enfocan en eventos placenteros, amistades y experiencias a pesar que deben confrontar con eventos como la muerte de seres queridos, la pérdida del estado activo asociado con la jubilación, deterioro de la salud, y la reducción de los ingresos (Lockenhoff \& Carstensen, 2004). Por otro lado, los adultos en la mediana edad se encuentran motivados para obtener ingresos incluso a expensas de su propio bienestar para obtener estabilidad económica y bienestar más adelante en la vida (Steptoe, Deaton, \& Stone, 2015). Es importante señalar que varios adultos mayores participan en diversos programas de servicios sociales en donde se promueve el envejecimiento activo o exitoso. El modelo de envejecimiento activo se basa en el concepto de ciclo vital con sus ganancias, estabilidad, y pérdidas en la edad madura que son asumidas de una forma adaptativa, y en el caso del adulto mayor estarán enfocados a la reestructuración de tiempo libre, el sentirse parte útil de la sociedad y sobre todo encontrar nuevas fuentes de apoyo social (Jiménez et al., 1999). La evaluación de impacto de dichos programas de asistencia o servicios sociales está fuera del alcance de este estudio.

Con este trabajo se intenta superar las limitaciones metodológicas de investigaciones anteriores planteando modelos de análisis factorial exploratorio, confirmatorio, y la utilización de matrices policóricas obedeciendo a la tendencia de adaptación y validación. Es substancial y existen cada vez más investigaciones que subrayan la importancia de la valoración de los aspectos psicométricos de los instrumentos.

Es importante señalar que el total de observaciones con las cuales se realizaron los análisis factoriales $(n=550)$ puede ser el origen de un sesgo muestral (Ten Berge \& Socan, 2004).

En conclusión, la versión en español de MHCSF (Keyes, 2009) es una herramienta apropiada para medir la salud mental y puede ser utilizada en el contexto ecuatoriano para la evaluación de la salud mental positiva de acuerdo con la fiabilidad y validez obtenidos en este estudio.

\section{Referencias}

Barrera Guzmán, M. L., y Flores- Galaz, M. M. (2015). Positive Mental Health, Construction of a Measurement Scale for Adults in Mexican Population. Revista Iberoamericana de Diagnóstico y Evaluación - e Avaliação Psicológica, 39(1), 22-33.

Blanco, A., y Diaz, D. (2005). El bienestar social: su concepto y medición. Psicothema, 17(4), 582-589.

Lima-Castro, S., Peña-Contreras, E., Cedillo-Quizhpe, C., y Cabrera-Vélez, M. (2017). Adaptación del Perfil PERMA en una muestra ecuatoriana. Eureka (Asunción) en Linea, 14, 69-83. Recuperado de http: //psicoeureka. com.py/sites/default/files/articulos/eureka-14-1-12.pdf

Butler, J., y Kern, M. L. (2016). The PERMA-Profiler: A brief multidimensionalmeasure of flourishing. International Journal of Wellbeing, 6(3), 1-48. doi:1.5502/ijw.v6i3.526

Brislin, R. W., Lonner, W. J., y Thorndike, E. M. (1973). Crosscultural research methods. New York: Wiley.

Carvalho, J. S., Salgado, P. N., Marquéz, P. A., y Maroco, J. (2016). Psychometric properties of the Mental Health Continuum-Short Form: A study of Portuguese speaking children youths. Journal of Child and Family Studies, (1) 1-14. doi:1.1007/s10826-016-0396-7 
Cicchetti, D. V. (1994). Guidelines, criteria, and rules of thumb for evaluating normed and standatized assesment in psychology. Psychological Assessment, 6(4), 284-29. doi:1.1037/1040-359.6.4.284

Chen FF, West SG \& Sousa KH.A (2006). Comparison of Bifactor and Second-Order Models of Quality of Life. Multivariate Behav Res.1;41(2):189-225. doi: 10.1207/ s15327906mbr4102_5

du Plessis, G. A., \& de Bruin, G. P. (2015). Using Rasch modelling to examine the international personality item pool (IPIP) values in action (VIA) measure of character strengths. Journal of Psychology in Africa, 25, 512-521.

Echeverría, G., Torres, M., Pedrals, N., Padilla, O., Rigotti, A. y Bitran, M. (2017). Validation of a Spanish Version of the Mental Health Continuum-Short Form Questionnaire. Psicothema, 29(1), 96-102. doi: http://doi.org/1.7334/ psicothema2016.3

Guillemin, F., Bombardier, C., y Beaton, D. (1993). Crosscultural adaptation of health related quality of life measures: Literature review and proposed guidelines. Journal of Clinical Ekpidemiology, 46, 1417-1432. doi: 1.1016/0895-4356(93)90142-N

Guo, C., Tomson, G., Guo, J., Li, X., Keller, C., y Friedrik, S. (2015). Psychometric evaluation of the Mental Health Continuum-Short Form (MHC-SF) in Chinese adolescents - a metodological study. Health and Quality of Life Outcomes, 13,198. doi: 10.1186/s12955-015-0394-2

Hu, L., y Bentler, P. M. (1999). Cut-off criteria for fit indexes in covariance structure analysis: Conventional criteria versus new alternatives. Structural Equation Modelling, 6,1-55. doi:1.1080/10705519909540118

Huppert, F. A., y So, T. T. (2013).Flourishing across Europe: Application of a new conceptual framework for defining well-being. Social Indicators Research, 110(3) 837-861. doi: 1.1007/s11205-011-9966-7.

Jovanović, Veljko. (2015). Structural validity of the Mental Health Continuum-Short Form: The bifactor model of emotional, social and psychological well-being. Personality and Individual Differences, 75, 154-159. doi: 10.1016/j.paid.2014.11.026

Jiménez, L., Lafuente, D., \& Hernández, B. (1999). Mayores: Calidad de vida y necesidades básicas. Rev Rol Enf, 22(11), 743-6.

Joshanloo, M., Wissing, M. P., Khumalo, I. P., y Lamers, S. M. (2013). Measurement invariance of the Mental Healt Continuum- Short Form (MHC-SF) across three cultural groups. Personality and Individual Differences, 55(7) $775-759$

Karaś, D., Cieciuch, J., y Keyes, C. L. ((2014). The Polish adaptation of the Mental Healt Continuum- Short Form (MHCSF). Personal and Individual Differences, 69, 104-109.

Keyes, C. (2002). The mental health continuum: from languishing of flourishing in life.Journal of Health and Social Behavior, 1, 207-222.Recuperado de: http://www. jstor.org/stable/3090197

Keyes, C. (2005). The subjective well-being of America's youth: Toward a comprenhensive assesment. Adolescent and Family Health, 4(1) 3-11. Recuperado de: https://www. researchgate.net/publication/232509662_The_Subjective_Well-Being_of_America's_Youth_Toward_a_Comprehensive_Assessment
Keyes, C. (2007). Promoting and protecting mental health as flourishing: A complementary strategyfor improving national mental health. American Psychologist, 62(2), 95-108. doi.org/1.1037/0003-066X.62.2.95

Keyes, C. (2009). Brief description of the Mental Health Continuum-Short Form (MHC-SF).Recuperado de: https:// www.aacu.org/sites/default/files/MHC-SFEnglish.pdf

Keyes, C. L., Corey, L., Shmotkin, D., y Ryff, C. D. (2002). Optimizing well-being: the empirical encounter of two traditions. Journal of Personality and Social Psychology, 1007.doi:1.1037/0022-3514.82.6.1007

Kokko, K., Korkalainen, A., y Lyyra, A. L. (2013). Structure and Continuity of Well-Being in Mid Adulthood: A longitudinal Study. Journal of Happiness Studies, 14(1), 99-114. doi: 1.1007/s10902-011-9318-y

Lamers, S., Glas, C., Westerhof, G., y Bolemeijer, E. (2011). Longitudinal Evaluation Of The Mental Health Continuun- Short Form (MHC-SF). Measurement Invariance Across Demographics, Physical Illnes, And Mental Illness. European Journal of Psychological Assessment. 28, 290-296. doi:1.1002/jclp.20741

Lima-Castro, S., Peña Contreras, E. K., Cedillo Quizphe, C., \& Cabrera Vélez, M. (2017). Adaptación del perfil perma en una muestra ecuatoriana. Eureka, 14(1), 1-166.

Lupano Perugini, M. L., de la Iglesia, G., Castro Solano, A. y Keyes, C. L. M. (2017). The Mental Health ContinuumShort Form (MHC-SF) in the Argentinean Context: Confirmatory Factor Analysis and Measurement Invariance. European Journal of Psychological Assessment, 13(1), 93-108. doi: 10.5964/ejop.v13i1.1163

Löckenhoff, C.E. y Carstensen, L.L. (2004). Socioemotional selectivity theory, aging, and health: The increasingly delicate balance between regulating emotions and making tough choices. Journal of Personality, 72, 1395-1424.

Petrillo, G., Capone, V., Caso, D., y Keyes, C. (2015). The Mental Healt Continuum-Short Form (MHC-SF) a measure of well-being in the Italian context. Social Indicators Research, 121(1) 291-312. doi: 1.1007/s11205-014-0629-3

Rhemtulla, M., Brosseau-Liard, P. y Savalei, V. (2012). When Can Categorical Variables Be Treated as Continuous? A Comparison of Robust Continuous and Categorical SEM Estimation Methods Under Suboptimal Conditions. American Psychological Association, 17(3), 354-373.

Organización Mundial de la Salud (2016). Salud mental. Temas de salud. Recuperado de: http://www.who.int/features/ factfiles/mental_health/es/

Steiger, J. H., y Lind, C. . (1980). Statistically based tests for the number of common. Annual meeting of the Psychometric Society. Congreso Anual de la Sociedad Psicométrica, Iowa, Estados Unidos.

Steptoe, A., Deaton, A., y Stone, A. A. (2015). Subjective wellbeing, health, and ageing. The Lancet, 385(9968), 640-648. doi:http://dx.doi.org/10.1016/S01406736(13)61489-0

Ten Berge, J.M.F., \& Socan, G. (2004). The greatest lower bound to the reliability of a test and the hypothesis of unidimensionality. Psychometrika, 69, 613-625.

Yañez, S., y Cárdenas, M. (2010). Estrategias de aculturación, indicadores de salud mental y bienestar psicológico en un grupo de inmigrantes sudamericanos en Chile. Revista Salud y Sociedad, 1(1) 51-7. Recuperado de: https:// dialnet.unirioja.es/descarga/articulo/4016251.pdf 